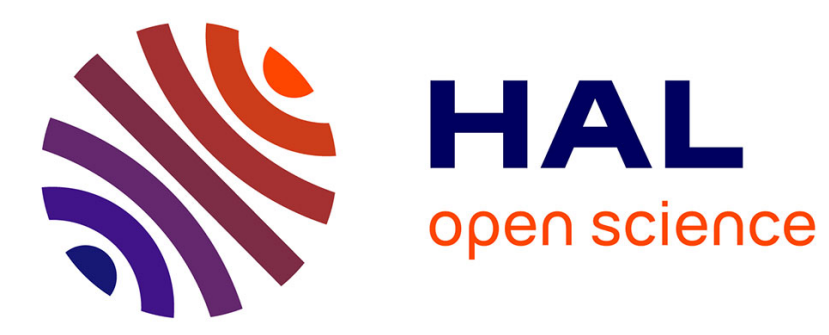

\title{
DYNAMICAL BEHAVIOR OF A NEODYMIUM DOPED FIBER LASER
}

D. Derozier, S. Bielawski, P. Glorieux

\section{To cite this version:}

D. Derozier, S. Bielawski, P. Glorieux. DYNAMICAL BEHAVIOR OF A NEODYMIUM DOPED FIBER LASER. Journal de Physique IV Proceedings, 1991, 01 (C7), pp.C7-367-C7-370. 10.1051/jp4:1991796 . jpa-00251039

\section{HAL Id: jpa-00251039 https://hal.science/jpa-00251039}

Submitted on 1 Jan 1991

HAL is a multi-disciplinary open access archive for the deposit and dissemination of scientific research documents, whether they are published or not. The documents may come from teaching and research institutions in France or abroad, or from public or private research centers.
L'archive ouverte pluridisciplinaire HAL, est destinée au dépôt et à la diffusion de documents scientifiques de niveau recherche, publiés ou non, émanant des établissements d'enseignement et de recherche français ou étrangers, des laboratoires publics ou privés. 


\title{
DYNAMICAL BEHAVIOR OF A NEODYMIUM DOPED FIBER LASER
}

\author{
D. DEROZIER, S. BIELAWSKI and P. GLORIEUX
}

Laboratoire de Spectroscopie Hertzienne, Université des Sciences et Techniques de Lille FA., Bât. P5, F-59655 Villeneuve d'Ascq cedex, France

Abstract: The dynamics of a doped fiber laser has been investigated under various conditions of pump modulation. Linear and nonlinear behaviors have been studied along two orthogonally polarized directions of the laser radiation. Antiphase phenomena have been observed between the two polarized radiations. A phenomenological model is proposed which takes all experimental effects into account.

In this paper, we provide an extensive description of the different dynamical regimes of a doped fiber laser under various conditions of pump modulation. In particular, the simultaneous exploration of the temporal evolution of output intensities of the two eigenstates of polarization provides a global description of the laser system. The first part presents the experimental set up and the linear behavior of the fiber laser. The nonlinear dynamics is investigated in the second part and the last part is devoted to a new simple phenomenological model in good agreement with the experimental results.

The active medium of the laser is a 5 meter long $300 \mathrm{ppm} \mathrm{Nd}^{3}+$ silica fiber doped with a core diameter of $5.8 \mu \mathrm{m}$ (the fiber is single mode at the laser operating wavelength: $1.08 \mu \mathrm{m}$ ). The laser cavity is limited by two dichroic mirrors transparent at the pump wavelength $(820 \mathrm{~nm})$ but with reflection coefficients $\mathrm{R} 1>99.5 \%, \mathrm{R} 2=95 \%$ at $1.08 \mu \mathrm{m}$. They are butt-coupled to the fiber and the pump radiation, emitted by a polarized single mode laser diode, is focused into the fiber through two microscope objectives. The two orthogonal polarizations of the laser radiation are optically separated and directed onto silicium photodiodes. A beam splitter is used to send a part of the polarized laser intensity in a monochromator for spectral analysis. Under typical operating conditions: $8 \mathrm{~mW}$ of the pump power are coupled into the fiber with an usual laser threshold of $2 \mathrm{~mW}$. When the pump power is twice the threshold value, the laser becomes strongly multimode with a wide $\operatorname{spectrum}\left(70 \mathrm{~cm}^{-1}\right)$ that may be shifted over $400 \mathrm{~cm}^{-1}$ between 1.06 and $1.10 \mu \mathrm{m}$.

When the spectrum center frequency is around $1.08 \mu \mathrm{m}$, we have investigated, using polarization optics, the evolutions of both the laser spectrum and the laser power versus the pump power for the two orthogonally polarized directions. The laser starts at the threshold pump power $P_{t h 1}$ and up to a second value $P_{t h 2}$, the laser radiation remains polarized along one particular direction. In this range, the laser response to a small temporal perturbation of the pump power is a single frequency damped oscillation 
(relaxation oscillations). When the pump power is further increased, a second polarization direction appears in the spectrum. Simultaneously to the appearance of this component narrower than the first one, the $\mathrm{CW}$ lasing characteristic of the first polarization exhibits a breaking in its slope. In these conditions, the relaxation oscillations are the sum of two damped oscillations with two different oscillation frequencies.

The relaxation frequency of the laser lies in the $10 \mathrm{kHz}$ range and modulation is easily achieved by varying the current injected in the pump laser diode. We have investigated the correlation between the radiation emitted at different wavelengths and in the two polarization states. We have observed the evolution of the responses of the laser on the two orthogonally polarized directions as the frequency of the modulation is swept near the relaxation oscillation resonance of the laser system. The modulation amplitude is kept constant and the instantaneous pump power remains always above threshold. The laser output intensities follow the modulation far from resonance. When approaching it, the system undergoes a cascade of period doubling bifurcations leading to chaos. The measurements are made simultaneously on the two orthogonal polarizations and we clearly observe antiphase dynamics on the time traces (Figure 1a). In the case of a $2 \mathrm{~T}$-periodic response, the maximun output intensity in one polarization direction corresponds to the small peak in the other one. The same phenomenum is observed on the nT-periodic signals and in the chaotic regime: large peaks in one direction are associated with small peaks in the other one. A third photodetector behind the monochromator is used to compare the total output intensity in one polarization with the same polarized intensity integrated on a narrow band of the spectrum. We don't observe significative phase differences between these two time traces. All the longitudinal modes in the same polarization state oscillate in phase but in antiphase with the longitudinal modes of the other state.

a

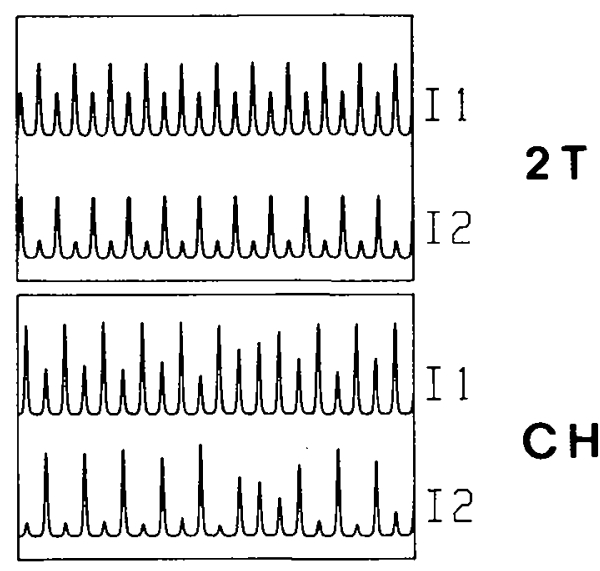

b

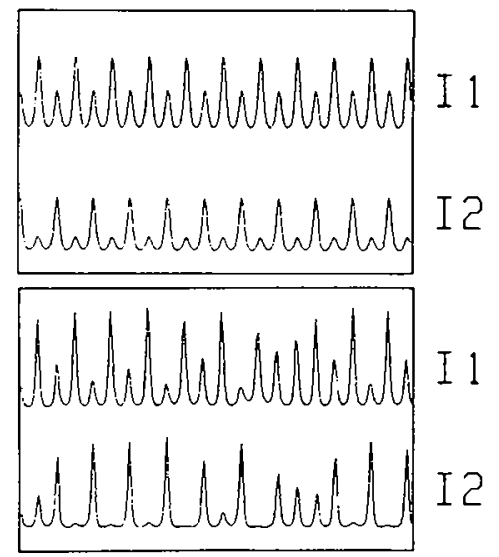

Eloure 1: Time dependence of the intensities 11,12 corresponding to the two polarization eigenstates for a $2 T$ and a chaotic regime. a: experimental, b: numerical results. 
The evolution of the laser dynamics reported in the series of recordings of Figure 1 a can be synthetized in a single bifurcation diagram using periodic sampling of the laser output intensity synchronously with the modulation [1]. With this technique, the sampling unit delivers a single-valued output when the response of the system is $T$-periodic, $n$ different values when its period is nT and scattered values for a chaotic response. Figure $2 a$ shows two examples of bifurcation diagrams when the modulation frequency is swept from low to high frequencies and vice-versa while the modulation amplitude is kept constant.
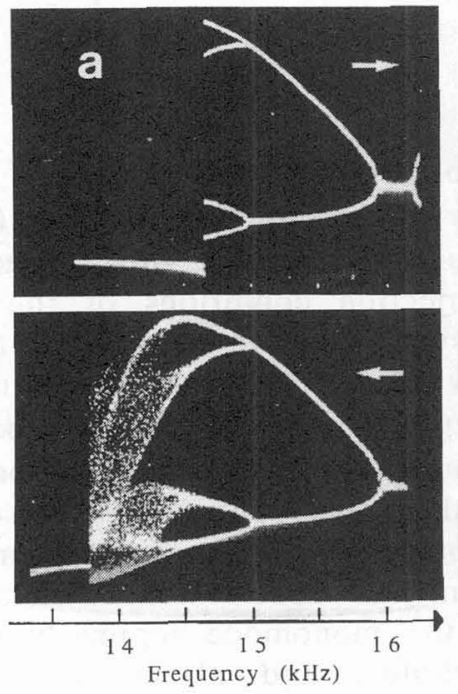

Eigure 2: Experimental ( $a$ ) and numerical $(b)$ bifurcation diagrams obtained for increasing and decreasing frequencies.

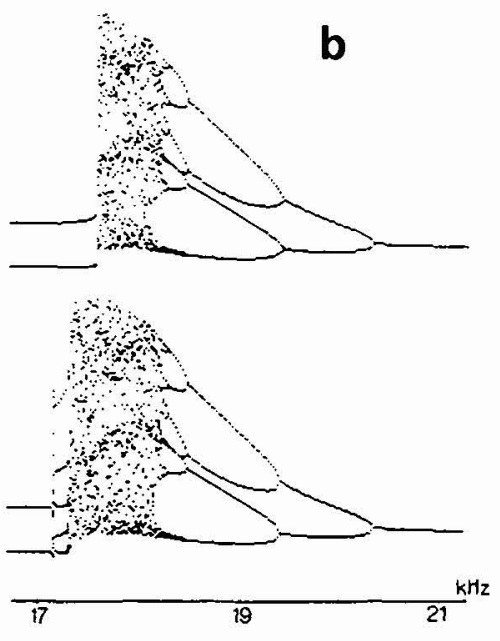

Far from resonance, the response is periodic and a clear period doubling cascade appears on the high frequency side when approaching the resonance: T-periodic at $16 \mathrm{kHz}, 2 \mathrm{~T}$-periodic from 15.9 to $15 \mathrm{kHz}$, 4T-periodic between 15 and $14.7 \mathrm{kHz}$. In the condition of a decreasing sweep, the chaotic region appears between 14.7 and $13.8 \mathrm{kHz}$ and it is suddenly interrupted at $13.8 \mathrm{kHz}$ by a $2 \mathrm{~T}$-periodic response while in the case of an increasing sweep, it is the $2 \mathrm{~T}$ periodic response which is suddenly interrupted by the $4 \mathrm{~T}$-periodic response. As there is no continuity between the branches of the chaotic region (resp. 4Tregion) and those of the $2 \mathrm{~T}$-periodic one, we can conclude that, there are two different attractors and that a boundary crisis leads to the disappearance of one of them. The boundary crisis is accompanied by generalized bistability between 14.7 and $13.8 \mathrm{kHz}$ since before the chaotic attractor disappears in the crisis, it coexists with one other stationnary regime of the laser [2].

In order to set up a phenomenological model of the fiber laser taking these effects into account in particular the two eigenfrequencies of the system and the antiphase phenomena, we can divide the amplifying medium into two classes of ions, each interacting strongly with one polarization eigenstate of the cavity. The two populations $\mathrm{N}_{1}, \mathrm{~N}_{2}$ are not only coupled to their corresponding 
polarizated intensities $I_{1}, I_{2}$ with coefficients $B_{1}, B_{2}$, but also to the other polarization via cross coupling parameters $\beta_{21} B_{2}$ and $\beta_{12} B_{1}$. For a guided laser, it is necessary to take into account the spontaneous emission. The spontaneous emission in one polarization state may be simply introduced in the equations, assuming that it is proportional to the gain in this polarization. The evolution of the intensities and the populations may be written:

$$
\begin{aligned}
& \dot{I}_{1}=B_{1}\left(N_{1}+\beta_{12} N_{2}\right) I_{1}-\kappa_{1} I_{1}+a\left(N_{1}+\beta_{12} N_{2}\right) \\
& \dot{I}_{2}=B_{2}\left(N_{2}+\beta_{21} N_{1}\right) I_{2}-\kappa_{2} I_{2}+a\left(N_{2}+\beta_{21} N_{1}\right) \\
& \dot{N}_{1}=\gamma\left(N_{01}\left(t-N_{1}\right)-B_{1} N_{1} I_{1}-B_{2} \beta_{21} N_{1} I_{2}\right. \\
& \dot{N}_{2}=\gamma\left(N_{02}\left(t-N_{2}\right)-B_{2} N_{2} I_{2}-B_{1} \beta_{12} N_{2} I_{1}\right.
\end{aligned}
$$

where $\kappa_{1}, \kappa_{2}$ are the cavity losses for the two polarizations, $\gamma$ the relaxation rate of the upper level of the laser transition, $\mathrm{N}_{01}(\mathrm{t}), \mathrm{N}_{02}(\mathrm{t})$ the intantaneous pumping terms of the two populations and a the spontaneous emission coefficient. If we remark that the injection conditions of the pump change the threshold values and the two intensity behaviors and if we assume that all the longitudinal modes are equivalents: we can perform the approximations $\kappa_{1}=\kappa_{2}, B_{1}=B 2=B$ and $\beta_{12}=\beta_{21}=\beta$ in the theoretical model. In theses conditions, it is only the instantaneous pumping terms which introduce a difference between the two set of longitudinal modes. The evolution of the steady states and the nature of the eigenvalues of the linearized system with the pump rate are the same as in the experimental observation: the laser is "off" below the first threshold, it operates in the monomode regime with one relaxation frequency between the two thresholds, and above the second threshold operates in the bimode regime with two relaxation frequencies. The experimental study of the variations of the two resonance frequencies in the bimode regime allows the determination of the laser parameters.

The numerical integration of the differential equations in presence of pump modulation has been carried out with the values of the parameters determined experimentally in order to test the model. The time dependences of the two intensities (figure $1 \mathrm{~b}$ ) are the same as the experimental ones, displaying also a spiking behavior, a period-doubling sequence when the frequency is swept and antiphase effects. The bifurcation diagrams obtained with the set of parameters corresponding to the experimental investigation of the fiber laser dynamics are presented in figure $2 \mathrm{~b}$. They display the same shape as the experimental ones. In particular, the chaotic region is delimited at high frequencies by a period-doubling cascade and at low frequencies by a boundary crisis.

To summarize, the observation of the onset of temporal instabilities in the fiber laser allows to propose a new phenomenological model in good agreement with the experimental results. To obtain more informations about the laser system, a frequency resolved study is necessary to take into account the multimode effects.

[1] D. Dangoisse, P. Glorieux and D. Hennequin, Phys. Rev. A 36 (1987) 4775.

[2] D. Derozier, S. Bielawski and P. Glorieux, Optics Comm. 83 (1991) 97. 